\title{
Epigenetic modification of RhoE expression in gastric cancer cells
}

\author{
JI CHEN, HAIJUN ZHOU, QIU LI, MENG QIU, ZHIPING LI, QIULIN TANG, MING LIU, YAJIE ZHU, \\ JUAN HUANG, NAN LANG, ZHEN LIU, YU DENG, SIYUAN ZHANG and FENG BI \\ Department of Medical Oncology/Laboratory of Signal Transduction and Molecular Targeted Therapy, \\ Cancer Center of West China Hospital, Sichuan University, Chengdu, Sichuan Province 610041, P.R. China
}

Received April 27, 2010; Accepted June 14, 2010

DOI: 10.3892/or_00001058

\begin{abstract}
RhoE is a unique member of Rho family of GTPases without detectable intrinsic GTPase activity. Our previous study showed that RhoE is a tumor suppressor gene and its expression is down-regulated in gastric cancer. However, the mechanism underlying the down-regulated expression of RhoE in gastric cancer has not been elucidated yet. In the present study, the effect of epigenetic modification on the RhoE expression in gastric cancer cells was investigated. The mRNA and protein expression of RhoE were detected by real-time RT-PCR and Western blotting, respectively. Results showed RhoE was significantly down-regulated in three gastric cancer cell lines. A promoter (2980 bp) of RhoE and its five truncated mutants were cloned into vector pGL-3Basic for the activity analysis by luciferase reporter assay. Treatment with trichostatin A, a histone deacetylation inhibitor, enhanced not only the activity of RhoE promoter, but also the mRNA and protein expression of RhoE in three gastric cancer cell lines, whereas treatment with 5-Aza-2'-deoxycytidine, a DNA methylation inhibitor, affected neither RhoE promoter activity nor RhoE expression. No synergistic effect was observed in cells treated with both drugs. Our results suggested that RhoE expression in gastric cancer cells was regulated by histone deacetylation, but not by DNA methylation, at the epigenetic level.
\end{abstract}

\section{Introduction}

Rho family proteins are members of the Ras superfamily of small GTP-binding proteins. They cycle between an active, GTP-bound conformation and an inactive, GDP-bound conformation (1). Their activity is controlled by guanine nucleotide exchange factors (GEFs), GTPase activating

Correspondence to: Dr Feng Bi, Department of Medical Oncology/Laboratory of Signal Transduction and Molecular Targeted Therapy, Cancer Center of West China Hospital, Sichuan University, No. 37, Guoxue Alley, Chengdu, Sichuan Province 610041, P.R. China

E-mail: bifeng@medmail.com.cn

Key words: RhoE, gastric cancer, promoter, histone deacetylation, DNA methylation proteins (GAPs), and GDIs (guanine nucleotide dissociation inhibitors). The representative members of Rho family are RhoA, Rac1 and Cdc42. They regulate multiple aspects of cell behaviour, including cell proliferation, invasion, migration and apoptosis (1-3). RhoE, together with Rnd1 and Rnd2 compose a subgroup of Rho GTPases, seems a unique member of Rho family (4). Unlike the typical Rho-family proteins, RhoE is found to be constitutively bound to GTP and shows no detectable GTPase activity (5).

RhoE is involved in the regulation of a wide spectrum of cellular functions (6). It plays an important role in regulating cell migration, cell cycle progression, cell transformation and promoting apoptosis (7-10). Previously, RhoE was reported to be down-regulated in prostate cancer tissue and cells (8). Our previous study demonstrated that compared with normal gastric tissue, RhoE expression was low in samples taken from gastric cancer patients (11). Therefore, RhoE may act as an important tumor suppressor gene in certain types of cancer.

Altered expression and/or activity of RhoE might be crucial to cancer progression and patient response to the therapy. However, the mechanism by which RhoE downregulation occurs in cancers has not been elucidated yet. To our knowledge, no mutation of RhoE gene has been reported in cancer types. Many researches have shown that epigenetic modification played an important role in gene expression regulation. Recently, two proteins in the Rho family have been reported to be regulated by epigenetic modification. One of two proteins is $\mathrm{RhoB}$, which was reported to act as a suppressor in many cancers (12). Interestingly, histone deacetylation modification, rather than DNA methylation, is the cause of its down-regulation in lung cancer (13). The other protein studied is Rnd1, which was also reported to be down-regulated by histone deacetylation in gastric cancer (14). RhoE shares $57 \%$ homology with RhoB, and together with Rnd1 belongs to the Rnd family. However, few studies investigate the effects of epigenetic modification on RhoE expression. In this study, we investigated whether epigenetic modification took part in down-regulation of RhoE expression in gastric cancer cells.

\section{Materials and methods}

Cell culture. Human gastric epithelial cell line (GES-1), human embryonic kidney cell line (HEK293) and gastric cancer cell lines (SGC7901, MKN45 and BGC823) were preserved in 
our laboratory. The GES-1, SGC7901, MKN45 and BGC823 cells were maintained in RPMI-1640 medium (Invitrogen, Carlsbad, CA, USA) supplemented with $10 \%$ fetal calf serum (FCS) (Gibco, Grand Island, NY, USA), penicillin (100 U/ml), and streptomycin $(100 \mu \mathrm{g} / \mathrm{ml})$ at $37^{\circ} \mathrm{C}$ in humidified air with $5 \% \mathrm{CO}_{2}$. HEK 293 cells were maintained in DMEM medium (Invitrogen) supplemented with 10\% FCS, penicillin (100 U/ $\mathrm{ml})$, and streptomycin $(100 \mu \mathrm{g} / \mathrm{ml})$.

Bioinformatics analysis of RhoE promoter. RhoE 5' regulatory sequence was obtained from NCBI Gene Bank database (http://www.ncbi.nlm.nih.gov/Genbank/). Putative transcription factor binding sites within RhoE promoter were analyzed by online software MatInspector (http://www.genomatix.de/ matinspector.html) and TFSEARCH (http://www.cbrc.jp/ research/db/TFSEARCH.html). The $\mathrm{CpG}$ Island was analyzed by online $\mathrm{CpG}$ island searcher (http://www . bioinformatics.org/sms2/cpg_island.html) and FirstEF (http://rulai.cshl.org/tools/FirstEF/).

Construction of promoter reporter plasmids. Genomic DNA of HEK293 cells was extracted using the universal genomic DNA extraction kit (Takara, Shiga, Japan) according to the manufacturer's instructions. A 3-kb human RhoE promoter fragment $(-2955-+25)$ relative to the translation initiation site (+1 ATG) was amplified via PCR from HEK293 cell genome using primers R1 and F1 (Table I). Primers were flanked by KpnI and HindIII restriction sites (restriction sites are underlined). The PCR products were gel-purified and sub-cloned into a TA cloning vector (Takara). After digestion with KpnI and HindIII enzymes (New England Biolabs, MA, USA), the insert was ligated into the promoterless luciferase reporter plasmid pGL-3Basic (Promega, Madison, WJ, USA), and then named pGL3-RhoEp-2980. A series of RhoE promoter 5' deletion constructs were amplified using the common reverse primer $\mathrm{F} 1$ and following sense primers: primer R2-R6 (Table I, restriction sites are underlined). The PCR products were digested by KpnI and HindIII enzymes and ligated into the promoterless luciferase reporter plasmid pGL-3Basic. New constructed plasmids were named pGL3RhoEp-2653, pGL3-RhoEp-2008, pGL3-RhoEp-1480, pGL3-RhoEp-1004, and pGL3-RhoEp-497, independently. All constructs were verified by restriction digestion and direct sequencing.

Cell transfection and luciferase reporter assay. Cells were plated at a density of $8 \times 10^{4}$ cells/well in 24-well plates for $24 \mathrm{~h}$ followed by transfection. Cells were transfected using lipofectamine 2000 (Invitrogen) according to the manufacturer's protocol. For cells in each well, $1 \mu \mathrm{g}$ of luciferase reporter constructs containing the 5' flanking fragments of human RhoE promoter or pGL-3 Basic/pGL-3 Control vector (as a negative/positive control) were co-transfected with $0.1 \mu \mathrm{g}$ of pRL-TK/pCMV-ß-galactosidase vector (Promega). PRL-TK/ pCMV-ß-galactosidase vector was used as control to detect transfection efficiency. Cells were harvested to analyze the basic RhoE promoter activity $24 \mathrm{~h}$ after transfection. To analyze the effect of epigenetic modification on RhoE promoter activity, the cells were treated with DNA methylation inhibitor 5-Aza-2'-deoxycytidine (5-Aza-dC; Sigma,
Table I. Sequence of PCR primers.

\begin{tabular}{|c|c|}
\hline $\begin{array}{l}\text { Amplification } \\
\text { sequence name }\end{array}$ & Primers $\left(5^{\prime}-3^{\prime}\right)$ \\
\hline RhoE promoter & F1: CCCAAGCTTTGGCTGGCTCTTCTCTCCTT \\
\hline pGL3-RhoEp-2980 & R1: CGGGGTACCGGGTCTGATCCCTACCTTAC \\
\hline pGL3-RhoEp-2653 & R2: CGGGGTACCGTGACTTCAGGCAGTTGGTT \\
\hline pGL3-RhoEp-2008 & R3: CGGGGTACCATCCATCCAAAGTCATACAG \\
\hline pGL3-RhoEp-1480 & R4: CGGGGTACCCATCAGTGCTTTTGACAACT \\
\hline pGL3-RhoEp-1004 & R5: CGGGGTACCTGTGGGGAGGTGCAACTAAG \\
\hline pGL3-RhoEp-497 & R6: CGGGGTACCGCATCTTTGGGATCTTCATG \\
\hline \multirow[t]{2}{*}{ RhoE } & F: ATAGAGTTGAGCCTGTGGGACAC \\
\hline & R: AGGGTCTCTGGTCTACTGATGTC \\
\hline \multirow[t]{2}{*}{ GAPDH } & F: CAAGGCCAACCGCGAGAA \\
\hline & R: CCCTCGTAGATGGGCACAGT \\
\hline
\end{tabular}

St. Louis, MO, USA) of various concentrations $(0.4,4$, and $10 \mu \mathrm{M})$ for $48 \mathrm{~h}$ or histone deacetylation inhibitor Trichostatin A (TSA; Sigma) of various concentrations (100, 250 and $500 \mathrm{nM}$ ) for $20 \mathrm{~h}$. To test the synergistic effect of 5-Aza$\mathrm{dC}$ and TSA on RhoE promoter activity, cells were treated with $4 \mu \mathrm{M} 5$-Aza-dC for $24 \mathrm{~h}$ followed by $250 \mathrm{nM}$ TSA for $20 \mathrm{~h}$. Then, cells were harvested in passive lysis buffer (Promega). Promoter activity was analyzed using DualLuciferase ${ }^{\circledR}$ reporter assay system (Promega) with a Synergy HT Multi-Mode microplate reader (BioTek, Winooski, Vermont, USA). Firefly luciferase activity was normalized to renilla luciferase activity in the untreated and 5-Aza-dC drug treated groups. As the activity of pRL-TK vector is influenced by TSA, firefly luciferase activity was normalized to $\beta$-galactosidase level in TSA treated groups by using the $ß$-galactosidase enzyme assay system (Promega) $(15,16)$.

5-Aza-2'-deoxycytidine and Trichostatin A treatment. To test the effect of epigenetic modification on RhoE expression, the SGC7901, MKN45 and BGC823 cells were seeded in 10-cm dishes at a density of $1 \times 10^{6}$ cells the day before drug treatment. Then, cells were treated with 5-Aza-dC of various concentrations $(0.4,4$ and $10 \mu \mathrm{M})$ for $72 \mathrm{~h}$ or TSA of various concentrations $(100,250$ and $500 \mathrm{nM})$ for $20 \mathrm{~h}$. To test the synergistic effect of 5-Aza-dC and TSA, the cells were treated with $4 \mu \mathrm{M} 5$-Aza-dC for $72 \mathrm{~h}$ followed by $250 \mathrm{nM}$ TSA for another $20 \mathrm{~h}$.

Real-time reverse transcription PCR. Total RNA $(1 \mu \mathrm{g})$ was extracted from control cells or cells treated with 5-Aza-dC, TSA or 5-Aza-dC plus TSA with Trizol (Invitrogen). RNA was reverse-transcribed into cDNA using random primers. The $\mathrm{RT}$ reaction product was amplified in a $25 \mu \mathrm{l}$ reaction with 2X SYBR premixed Ex Taq (Takara). Primers for RhoE and GAPDH were listed in Table I. The reactions were carried out in a 96-well plate, and PCR amplification protocol was: 
A

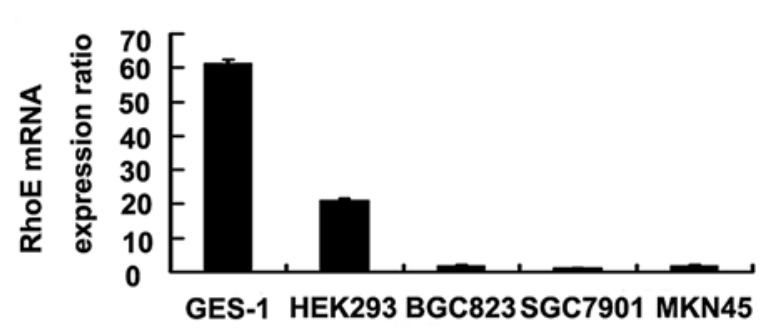

B

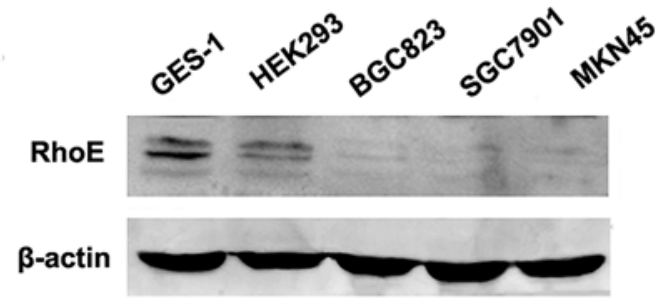

Figure 1. Expression of RhoE in different cell lines. RhoE expression in GES-1 cells, HEK293 cells, and three gastric cancer cell lines (BGC823, SGC7901 and MKN45) were examined by real-time RT-PCR and Western blotting. $\beta$-actin was used as a control. Expression of RhoE was decreased at both RNA (A) and protein (B) levels in all three gastric cancer cell lines as compared to GES-1 cells and HEK293 cells. Data were expressed as means \pm SD of three independent experiments.

$95^{\circ} \mathrm{C}$ for $1 \mathrm{~min}$ and 40 cycles of amplification at $95^{\circ} \mathrm{C}$ for $10 \mathrm{sec}, 55^{\circ} \mathrm{C}$ for $30 \mathrm{sec}$ and $72^{\circ} \mathrm{C}$ for $30 \mathrm{sec}$ in an iCycler iQ machine (Bio-Rad, Hercules, CA, USA), and melting curve analysis was performed immediately after amplification. The $2^{-\Delta \Delta C t}$ method was used to calculate the relative fold difference in the RhoE mRNA expression between all treated cell lines and control cell lines. Two-fold increased or decreased expression was considered significant (17).

Western blotting. Cells with or without treatment were washed with cold PBS three times and lysed in lysis buffer (10 mmol/1 Tris-HCl, pH 7.4, 1\% TritonX-100, $1 \mathrm{mM}$ EGTA, $1 \mathrm{mM}$ EDTA, $100 \mathrm{mmol} / \mathrm{l} \mathrm{NaCl}, 10 \%$ glycerol, $0.1 \%$ SDS, and $0.5 \%$ deoxycholate) containing protease inhibitor cocktail (Sigma) on a rotating shaker for $30 \mathrm{~min}$. Nuclei and unlysed cells were removed by centrifugation at 13,000 rpm for $30 \mathrm{~min}$ at $4^{\circ} \mathrm{C}$. Protein concentrations were determined using the BCA protein assay (Pierce, Rockford, IL, USA). Equal amounts of total protein were separated by $12 \%$ SDSPAGE and transferred to polyvinylidene fluorid membranes (Millipore, Billerica, MA, USA). Then, the membranes were washed and blocked with 5\% non-fat milk in PBS for $2 \mathrm{~h}$ followed by incubation with mouse anti-RhoE monoclonal antibody (1:200, Upstate, Lake Placid, NY, USA) or mouse anti- $\beta$-actin polyclonal antibody $(1: 1000$; Santa Cruz Biotechnology, Santa Cruz, CA, USA) at $4^{\circ} \mathrm{C}$ overnight. After extensive washing, the membranes were incubated with an IRDye $800 \mathrm{cw}$ fluoresce donkey anti-mouse secondary antibodies (1:10000; Li-Cor, Lincoln, NB, USA) for $2 \mathrm{~h}$ at room temperature in an opaque cassette. Finally the membranes were washed and immunoreactivity was detected using an Odyssey infrared fluorescence scanner (Li-Cor). Brand intensities were analyzed by using the Odyssey infrared image system (Li-Cor).
Statistic analysis. One-way ANOVA was used for statistical analysis. Data were expressed as means $\pm \mathrm{SD}$, and a value of $\mathrm{P}<0.05$ was considered statistically significant. The Statistic Package for the Social Sciences software program (version 13.0, SPSS, Chicago, IL, USA) was used for statistic analysis. All data were confirmed by at least three independent experiments.

\section{Results}

Expression of RhoE in different cell lines. In this experiment, RhoE expression in the human gastric epithelial cell line (GES-1 cells), human embryonic kidney cell line (HEK293 cells), and other three gastric cancer cell lines (BGC823, SGC7901, and MKN45 cells) was detected by real-time RTPCR and Western blotting, respectively. The results demonstrated that the expression of RhoE was decreased at both mRNA and protein levels in all three gastric cancer cell lines as compared to GES-1 cells and HEK293 cells (Fig. 1A and B), which was consistent with our previous observations.

Analysis of RhoE promoter. The transcription initiation site of RhoE was identified and verified though 5'RACE experiment by Ongusaha and his colleagues (18). It is located at $-249 \mathrm{bp}$ upstream from the ATG translation start codon $(+1)$. Computer-based analysis of RhoE promoter $(-2955-+25$ relative to the translation initiation site) with online software identified several potential transcription factor binding motifs in the promoter region, including transcription factors $\mathrm{p} 53$, AP-1, E2F, and MZF. However, no CpG island was found by online $\mathrm{CpG}$ island searchers in RhoE promoter region. Two inverted CCAAT boxes were found to be located in the RhoE promoter region (Fig. 2). The inverted CCAAT box was reported to be critical for the induction of RhoB promoter activity following TPX-mediated inhibition of HDAC (histone deacetylases) (16). To determine the critical region of RhoE promoter required for transcription activity, the 5' flanking region $(-2955-+25$ relative to the translation initiation site +ATG) was cloned and inserted into the upstream of a luciferase reporter plasmid pGL-3Basic vector. A series of reporter plasmids containing various lengths of RhoE promoter region were successfully constructed. RhoE promoter activity was analyzed as described in Materials and methods. The results showed that positive control plasmids pGl-3Control had the highest promoter activity $(62.3 \pm 5.5)$, and negative control plasmids pGl-3Basic did not present promoter activity. Luciferase reporter pGL3-RhoEp-2980 had the highest promoter activity (15.3 \pm 0.57$)$ among all RhoE promoters in HEK293 cells. RhoE promoter activity was reduced when RhoE promoter was truncated from -2980 to -2653: pGL3RhoEp-2653 (9.67 \pm 0.58$)$, from -2008 to -1480 : pGL3-

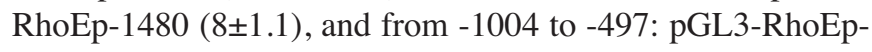
$497(7.3 \pm 1.15)$. Promoter activity was reversed back to a high level when RhoE promoter was truncated from -2653 to -2008: pGL3-RhoEp-2008 (13.3 \pm 0.56$)$ and from -1480 to

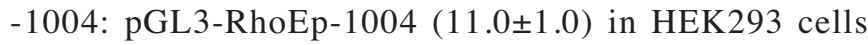
(Fig. 3A). The basic promoter activity of pGL3-RhoEp-2980 in the GES-1, HEK293, and other three gastric cancer cell lines was also analyzed. The promoter activities of pGL3RhoEp-2980 in different cells lines were: GES-1 (20 \pm 0.87$)$, 
GATA

-571 AGATAAGCCCCTATTAAACTATATTTAAAGGGATTGTAGT

p53

-531 TCAAATCAGTATGCACAGATACCAGTTTCCCACCTTGCCC -491 TGTGGACTTGTCAGCAAAGCATCTTTGGGATCTTCATGTT

-451 GTCTAAGAGCCTTGGTCTAAAATAGATCCCGAATGGGCAC -411 CCTAGAGGGCGCACTGCTTACATTTTCGGCATGCAACGCC ZF5 MZF

-371 AACAGCGCTCGCTCCCAGGCTCCATAAAAGGGGAGGAGGC -331 AGATCAGTTTCCTCCTCTCATTGAGAAAGAAGAGGATTGG E2F

-291 AAGGCCGGGCGTATAAAGCCGCGCAGAGAGCGTGAAACAA transcription start site

-251 AGCAGtcggctcGgAATtGgACtTGgGAGgCGCGgTGAGG AP1

-211 AGTCAGGCTTAAAACTTGTTGGAGGGGAGTAACCAGCCTG

-171 CTCCTCTCGCTCTCCTCCTCGTCTGCGCCGCGTTTCAGAG

-131 GTTGCCCATCAGCCTTGTGATTTATTTTTATATCTGCTTT

-91 TTATAAAGAGAGAAATATATATATATATATATATTTTTTT

-51 TTTCTTCTTAAGAGAAAATTCCTGTTCCAAGAGAAAATA

trans/ation start site

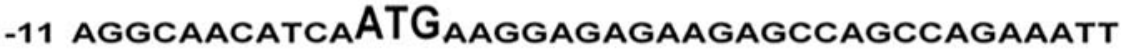

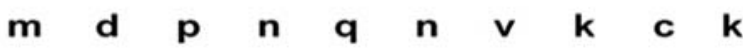

+31 ATCCAGCAAATCTATCATGGATCCTAATCAGAACGTGAAA

+71 TGCAAGATAGTTGTGGTGGG

$\begin{array}{lllllll} & \text { I } & h & \boldsymbol{h} & \mathbf{f} & \mathbf{a} & \mathbf{k}\end{array}$

Figure 2. Bioinformatics analysis of 5' upstream regulatory region of human RhoE gene. A 642 bp DNA sequence of 5' upstream regulatory region of RhoE gene is presented. The transcription initiation site (bold italic) of RhoE gene is located at -249 nucleotides upstream from the ATG translation start codon (+1) (bold). The amino acid sequence is shown in small letters. Computer-based analysis of RhoE promoter identified several potential transcription factor binding sites (underlined) located in the promoter region. Two inverted CCAAT boxes were also found to be located in the RhoE promoter region (grey box). No CpG island was found in RhoE promoter region.

HEK $293(15.3 \pm 0.58)$, BGC823 (3.0 \pm 0.02$),$ SGC790 1

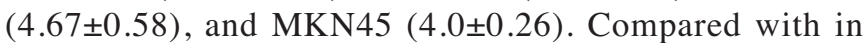
GES-1 and HEK293, pGL3-RhoEp-2980 promoter activity was markedly lowered in the three gastric cancer cell lines $(\mathrm{P}<0.05)$ (Fig. 3B).

Effect of 5-Aza-dC and/or TSA on the RhoE promoter activity in three gastric cancer cell lines. Effect of DNA methylation inhibitor 5-Aza-dC and/or the histone deacetylase inhibitor TSA on luciferase reporter pGL3-RhoEp-2980 in gastric cancer cell lines (BGC823, SGC7901, and MKN45 cells) was further investigated. Compared with untreated cells, RhoE promoter activity was induced by TSA $(100,250$, and $500 \mathrm{nM})$ treatment in a dose-dependent manner in BGC823 cells (3.1-, 4.2-, and 4.7-fold, respectively), SGC7901 cells (1.6-, 2-, and 2.2-fold, respectively), and MKN45 cells (1.9-, 2.9- and 3.3-fold, respectively) (Fig. 4A) $(\mathrm{P}<0.05)$. However, the RhoE promoter activity was not significantly changed after treatment with 5 -Aza-dC $(0.4,4$, and $10 \mu \mathrm{M})$ in the three gastric cancer cell lines (Fig. 4B) $(\mathrm{P}>0.05)$. Compared with TSA $(250 \mathrm{nM})$ treated cells, no synergistic effect was detected in the three gastric cancer cell lines after combined treatment with 5 -Aza-dC $(4 \mu \mathrm{M})$ for $24 \mathrm{~h}$ followed by TSA (250 nM) for $20 \mathrm{~h}$ (Fig. 4A) (P>0.05).
Effect of 5-Aza-dC and/or TSA on the expression of RhoE in three gastric cancer cell lines. To investigate the epigenetic modification on RhoE expression in gastric cancer cells, the mRNA and protein expression of RhoE were also analyzed by real-time RT-PCR and Western blotting, respectively, after treatment with 5-Aza-dC and/or TSA. Compared with untreated cells, the RhoE expression was profoundly increased in a dose-dependent manner in the TSA $(0,100,250$, and $500 \mathrm{nM}$ ) treated gastric cancer cells at both RNA (Fig. 5A-C) $(\mathrm{P}<0.05)$ and protein levels (Fig. 6A-F) $(\mathrm{P}<0.05)$. On the contrary, treatment with 5 -Aza-dC of 0.4 to $10 \mu \mathrm{M}$ failed to change the RhoE expression in the gastric cells at both mRNA (Fig. 5A-C) $(\mathrm{P}>0.05)$ and protein levels (Fig. 6A-F) $(\mathrm{P}>0.05)$. Compared with the TSA treated gastric cancer cells $(250 \mathrm{nM})$, no synergistic effect was observed in the gastric cancer cells treated with 5-Aza-dC $(4 \mu \mathrm{M})$ for $72 \mathrm{~h}$ followed by TSA $(250 \mathrm{nM})$ for $20 \mathrm{~h}$ by real-time RT-PCR (Fig. 5A-C) (P>0.05) and Western blot analysis (Fig. 6A-F) $(\mathrm{P}>0.05)$. These results were consistent with those in the promoter activity analysis.

\section{Discussion}

RhoE is a small GTPase protein which plays an important role in tumorigenesis. Since all the RhoE protein is in a 
A
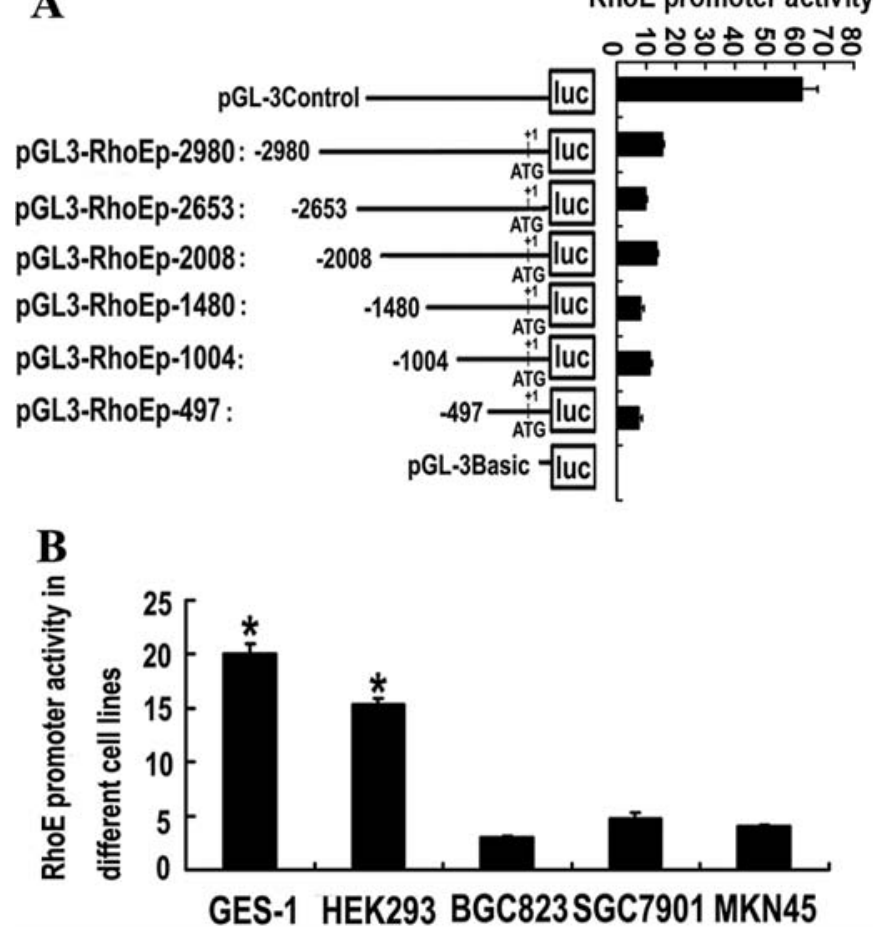

Figure 3. Luciferase reporter assay of RhoE promoter characteristics. (A) Activity of different truncation constructs of RhoE promoter in HEK293 cells. A series of reporter plasmids containing various lengths of RhoE promoter region were co-transfected with pRL-TK plasmids into the HEK 293 cells, and firefly luciferase activity was normalized to renilla luciferase activity. The schematic representation of RhoE promoter truncation constructs is shown on the left. The activity of RhoE promoter for each truncated constructs is shown on the right. All experiments were performed in triplicates. Data are presented as means $\pm \mathrm{SD}(\mathrm{n}=3)$. (B) Analysis of RhoE promoter activity in GES-1 cells, HEK293 cells, and three gastric cancer cell lines (BGC823, SGC7901, and MKN45) by luciferase reporter assay. RhoE promoter activity was lower in the three gastric cancer cell lines when compared with HEK293 and GES-1 cells $\left({ }^{*} \mathrm{P}<0.05\right)$. All experiments were performed in triplicate. Data are presented as means \pm $\operatorname{SD}(n=3)$.

GTP-bound active status, the expression of RhoE quite closely represents its activity. Aberrant expression of RhoE has been observed in several tumors. Although studies showed that RhoE was increased in pancreatic tumors (19) and non-small cell lung cancer (NSCLC) (20), RhoE expression was found to be significantly reduced or nonexistent in prostate cancer (8) and the U87 human glioblastoma cell line (9). In a previous study, we also found RhoE was down-regulated in gastric cell lines and gastric cancer tissue (11). Enhanced RhoE expression also affects the malignant phenotypes of cancer. In prostate cancer cell, exotic expression of RhoE induced cell cycle arrest and apoptosis (8). Overexpression RhoE in the U87 human glioblastoma cell line can inhibit cell proliferation and promote apoptosis (9). Therefore, RhoE may function as a tumor suppressor in these cancers. The understanding of the mechanism for RhoE down-regulation will be important for the development of future diagnostic and therapeutic strategies for clinical application.

Previous studies demonstrated that many factors could increase RhoE expression under different situations. RhoE expression was increased in cells after treatment with different
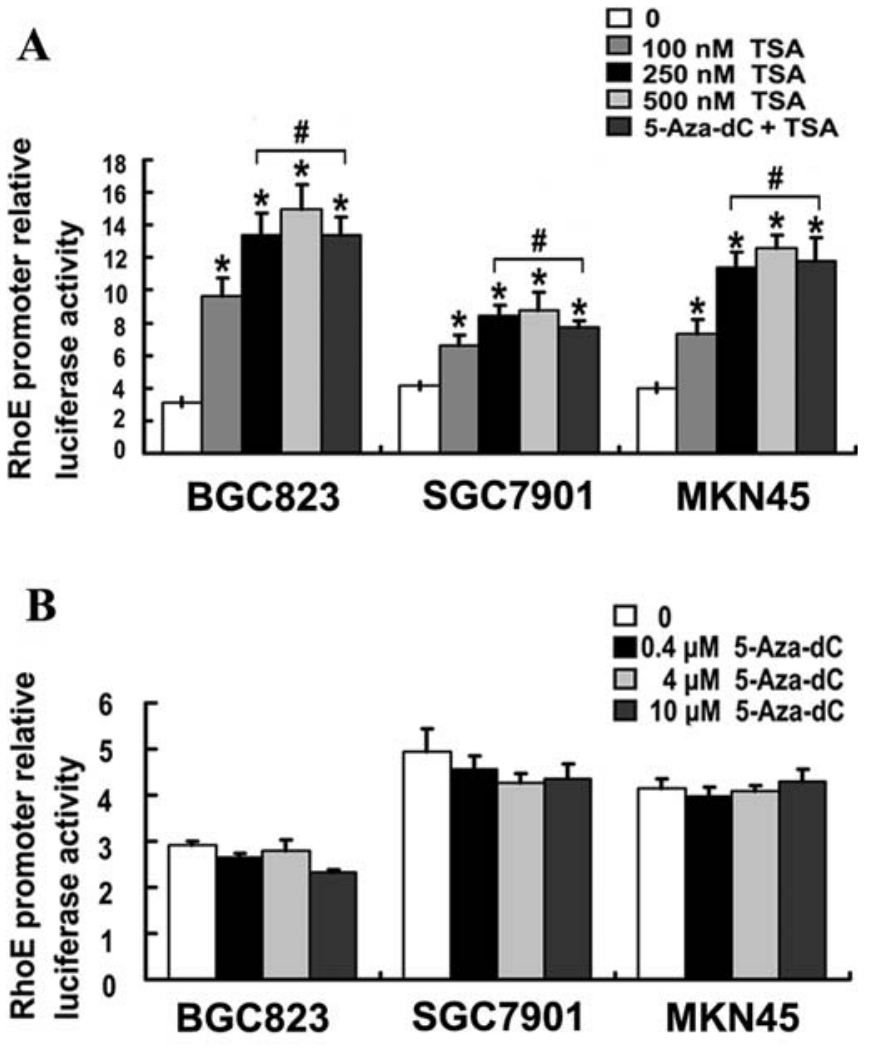

Figure 4. Effect of TSA and/or 5-Aza-dC on the RhoE promoter activity in three gastric cancer cell lines. (A) RhoE promoter activity changed in three gastric cancer cell lines after treatment with TSA and 5-Aza-dC plus TSA. Firefly luciferase activity was normalized to B-galactosidase level. Compared with untreated cells, RhoE promoter activity was increased in a dose-dependent manner following TSA treatment in the three gastric cancer cell lines $\left({ }^{*} \mathrm{P}<0.05\right.$ vs control). Compared with TSA $(250 \mathrm{nM})$ treated cells, no synergistic effect on the RhoE promoter activity was detected in the three cell lines after treatment with 5 -Aza-dC $(4 \mu \mathrm{M})$ followed by TSA $(250 \mathrm{nM})$ ( $\left.{ }^{\#}>0.05\right)$. (B) RhoE promoter activity was changed in the three gastric cancer cell lines after treatment with 5-Aza-dC. Firefly luciferase activity was normalized to renilla luciferase activity. RhoE promoter activity did not change after treatment with 5 -Aza-dC of various concentrations $(\mathrm{P}>0.05)$. Experiments were performed in triplicate. Data are presented as means \pm SD $(n=3)$. Statistical significance was determined by one-way ANOVA.

DNA damaging agents $(10,18)$ or UVB irradiation (21). RhoE expression was also induced through the Raf pathway in Madin-Darby canine kidney (MDCK) cells (22), and by platelet-derived growth factor (PDGF) in fibroblasts cells (23) or by hepatocyte growth factor in Swiss 3T3 or MDCK cells (24). Ongusaha and his colleagues proved RhoE was a direct p53 targeted gene and p53 played an important role in the up-regulated RhoE expression by DNA damaging agents (18). A previous study also demonstrated that phosphorylation of RhoE could increase its stability and prevent it from degradation by ubiquitin/proteasome (25). However, little is known about the mechanism in the down-regulation of RhoE in cancer. RhoE was reported to be down-regulated by oestrogens (26) or macrophage inhibitory cytokine-1 (27) in prostate cancer cells. However, the specific mechanism has not been elucidated and the reason for RhoE downregulation in human gastric cancer is still unknown. In order to find out the mechanism in RhoE down-regulation in human gastric cancer, bioinformatic analysis of RhoE promoter was performed. Results showed that no $\mathrm{CpG}$ island 
A

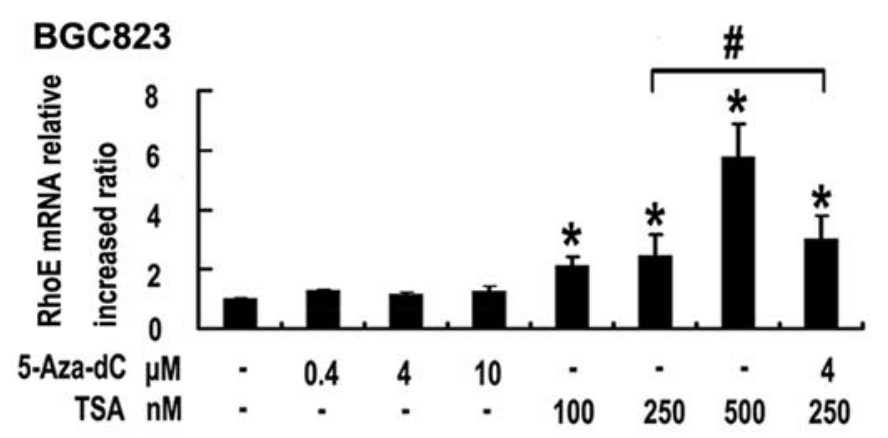

B
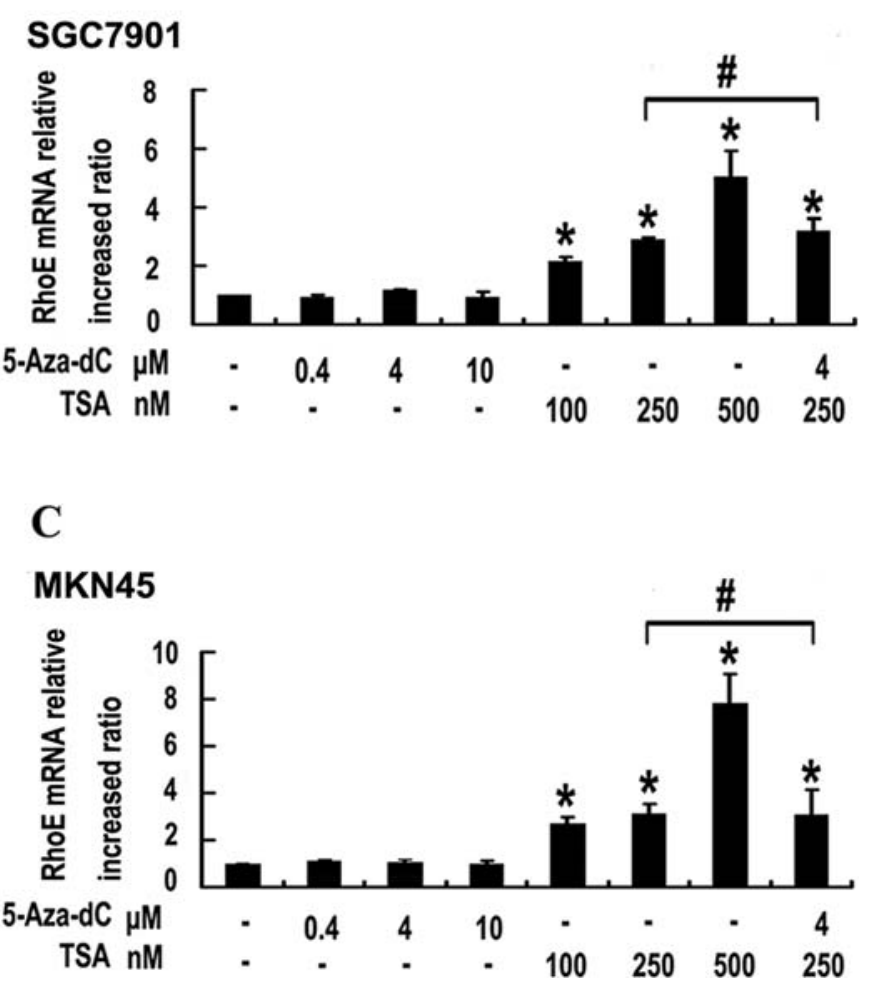

Figure 5. Effect of TSA and/or 5-Aza-dC on RhoE mRNA expression in three gastric cancer cell lines. The fold change of RhoE mRNA expression in BGC823 cells (A), SGC7901 cells (B), and MKN45 cells (C) after treatment with 5-Aza-dC and/or TSA are presented. GAPDH mRNA expression was used as control. Experiments were performed in triplicate. Data are presented as means $\pm \mathrm{SD}(\mathrm{n}=3)$. Statistical significance was determined by one-way ANOVA, ${ }^{*} \mathrm{P}<0.05$ vs. control; ${ }^{\#} \mathrm{P}>0.05$ : 5 -Aza-dC $(4 \mu \mathrm{M})$ plus TSA $(250 \mathrm{nM})$ treated cells vs. TSA $(250 \mathrm{nM})$ treated cells.

was found in RhoE promoter region, but several transcription factors were found to be likely bound to the RhoE promoter. Two inverted CCAAT boxes were also found in the RhoE promoter region. The inverted CCAAT box is critical for the induction of RhoB promoter by TPX (a HDAC inhibitor) in lung cancer cell (16). Further studies are needed to elucidate the role of these transcription factors and the inverted CCAAT boxes in regulating RhoE expression.

To find out the most critical component of RhoE promoter region in transcription, a series of reporter plasmids containing various lengths of RhoE promoter region were generated. After testing different truncations of RhoE promoter, we found pGL3-RhoEp-2980 had the highest promoter activity in HEK293 cells, and the activity was reduced when RhoE promoter was truncated from -2980 to $-2653,-2008$ to -1480 and -1004 to -497 . Interestingly, RhoE promoter activity was reverted back to a high level when it was truncated from -2653 to -2008 and -1480 to -1004 . These findings suggested the binding sites of some transcription factors negatively regulating RhoE expression might be located in these regions. Further studies are needed to identify these important transcription factors and their specific binding sites in RhoE promoter. RhoE promoter activity in different cell lines was also analyzed. Compared with GES-1 and HEK293 cells, RhoE promoter activity was markedly lowered in the three gastric cancer cell lines. These results were consistent with the reduced expression of RhoE in these three gastric cancer cell lines.

Besides genetic changes, epigenetic modification of genomic DNA is another important cause of altered gene expression in cancer cells. The most common epigenetic modifications in gene silencing are DNA methylation and histone deacetylation. Expression of many tumor suppressor genes are down-regulated by epigenetic modification during the tumorigenesis (28). Previous studies have shown that silencing of tumor suppressor genes by epigenetic modification plays an important role in gastric cancer development $(29,30)$. However, little is known about the effect of epigenetic modification on RhoE expression in gastric cancer.

In this study, we delineated the roles of histone deacetylation and DNA methylation in the regulation of RhoE expression in gastric cancer cell lines. RhoE promoter activity and expression levels after treatment with 5-Aza-dC and/or TSA in three gastric cancer cells were determined. The results showed, in gastric cancer cell lines, TSA, a histone deacetylation inhibitor, enhanced not only the RhoE promoter activity, but also the expression of RhoE both at mRNA and protein levels. However, 5-Aza-dC, a DNA methylation inhibitor, affected neither promoter activity nor RhoE expression. Furthermore, no synergistic effects were observed in cells treated with both drugs. These results suggested that, rather than DNA methylation, histone deacetylation might be the primary mechanism in modulating epigenetic transcriptional silencing of RhoE in these three gastric cancer cell lines. However, the mechanisms in the regulation of gene expression are complicated. The epigenetic mechanism for tumor suppressor gene silencing in cancer is not completely understood. A large number of reports demonstrate that DNA methylation and histone deacetylation have synergistic effects on gene expression.

Tumor suppressor genes such as MLH1, TIMP3, CDKN2B (31), are regulated by both DNA methylation and histone modification. However, our results, as well as those of other studies, showed some gene expression was regulated by only one type of epigenetic modification. For example, APC, P16, and RASSF1A (32) gene were reported to be regulated by only DNA methylation, while RhoB, CAR (33) and RhoE gene were regulated by histone deacetylation. In the case of RhoE, no $\mathrm{CpG}$ island was found in its promoter. However, the promoter region of CAR (33) and RhoB (13) contains CpG islands, and DNA methylation still does not seem to play a role in controlling their gene expression. The reason 
A

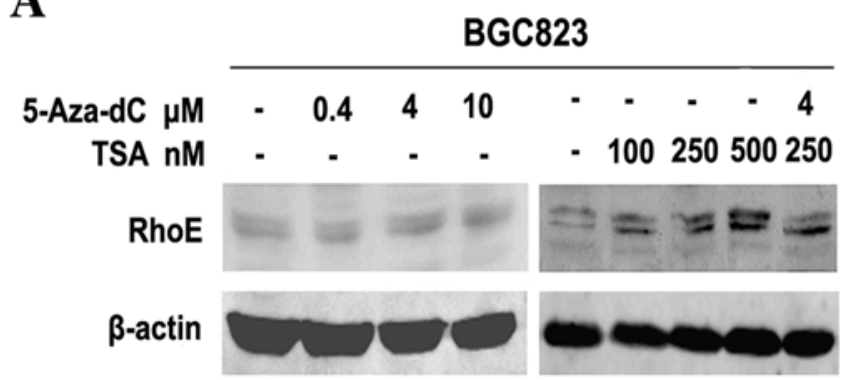

B

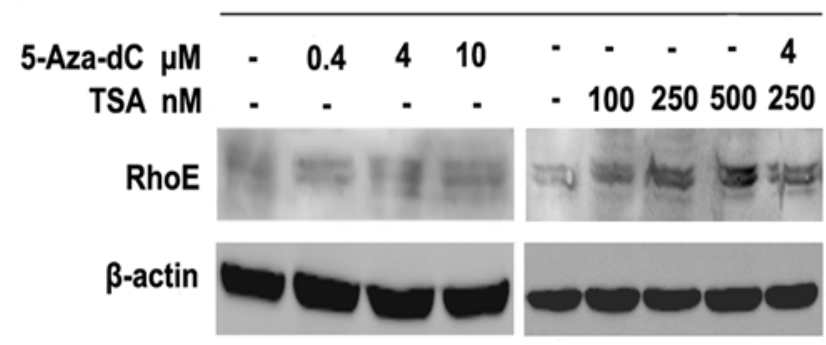

C

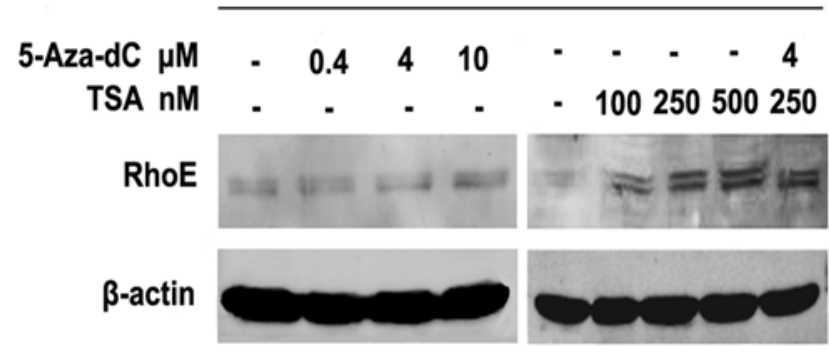

D $\mathrm{BGC} 823$

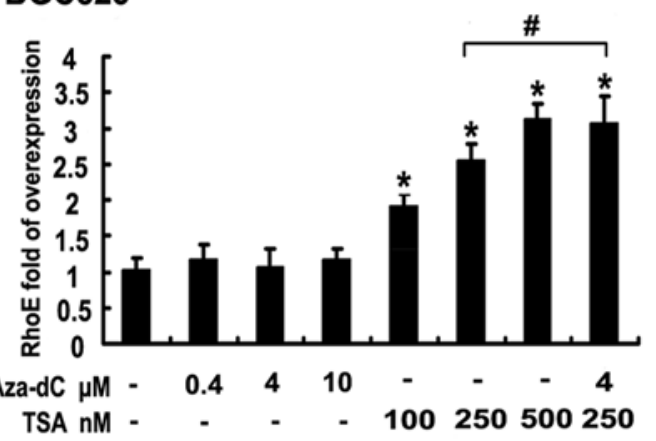

\section{E SGC7901}

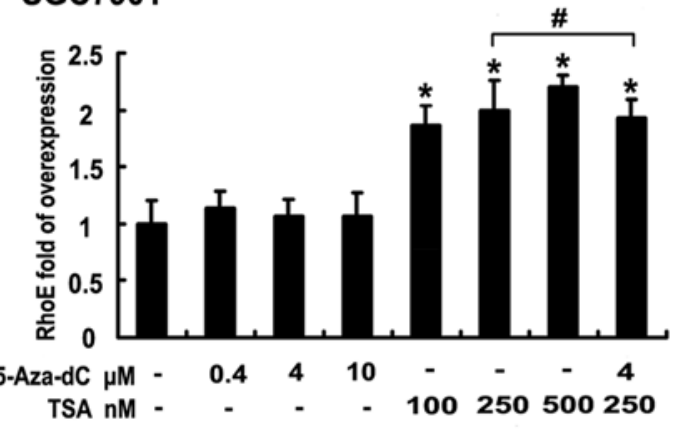

F

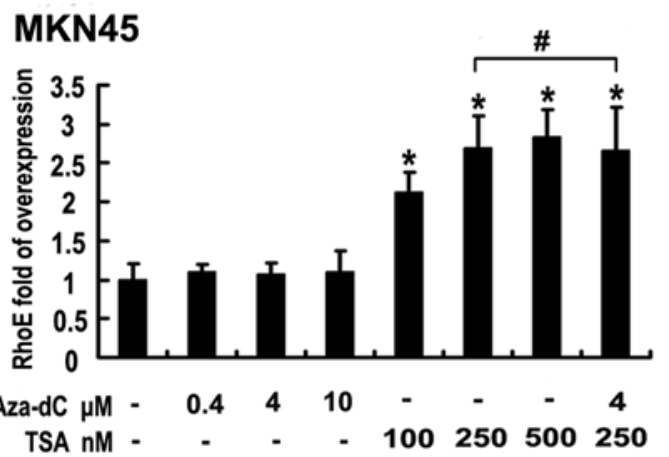

Figure 6. Effect of TSA and/or 5-Aza-dC on RhoE protein expression in three gastric cancer cell lines. RhoE protein expression was changed in BGC823 cells (A), SGC7901 cells (B), and MKN45 cells (C) after the treatment with 5-Aza-dC and/or TSA. B-actin protein expression was used as control. The fold change of RhoE protein expression after treatment in BGC823 cells (D), SGC7901 cells (E), and MKN45 cells (F) was quantified by Odyssey infrared image system. Experiments were performed in triplicate. Statistical significance was determined by one-way ANOVA. $\mathrm{P}<0.05$ vs. control; ${ }^{*} \mathrm{P}>0.05$ : $5-\mathrm{Aza}-\mathrm{dC}(4 \mu \mathrm{M})$ plus TSA $(250 \mathrm{nM})$ treated cells vs. TSA treated cells $(250 \mathrm{nM})$.

for this phenomenon is poorly understood. Further studies are needed to find out which factors determine the epigenetic modification process that regulates gene expression.

In summary, we analyzed the promoter characteristics of RhoE, and found that RhoE down-regulation in gastric cancer cell lines was modulated by histone deacetylation rather than DNA methylation at the epigenetic level. We hypothesize that the reversal of RhoE expression by HDAC inhibitors may be of therapeutic interest in gastric cancer.

\section{Acknowledgements}

This study was supported by the National Natural Science Foundation of China (grant no. 81071640). We thank Professor Yi Zheng for reviewing and proof-reading the manuscript.
We would also like to thank Mrs. Peiling Cai, Li Xu, and Xiangzheng Chen for helpful discussions and technical assistance.

\section{References}

1. Van Aelst L and C D'Souza-Schorey: Rho GTPases and signaling networks. Genes Dev 11: 2295-2322, 1997.

2. Ridley AJ and Hall A: The small GTP-binding protein rho regulates the assembly of focal adhesions and actin stress fibers in response to growth factors. Cell 70: 389-399, 1992.

3. Etienne-Manneville S and Hall A: Rho GTPases in cell biology. Nature 420: 629-635, 2002.

4. Foster R, Hu KQ, Lu Y, Nolan KM, Thissen J and Settleman J: Identification of a novel human Rho protein with unusual properties: GTPase deficiency and in vivo farnesylation. Mol Cell Biol 16: 2689-2699, 1996. 
5. Garavini H, Riento K, Phelan JP, McAlister MS, Ridley AJ and Keep NH: Crystal structure of the core domain of RhoE/Rnd3: a constitutively activated small G protein. Biochemistry 41 6303-6310, 2002.

6. Chardin P: Function and regulation of Rnd proteins. Nat Rev Mol Cell Biol 7: 54-62, 2006.

7. Guasch RM, Scambler P, Jones GE and Ridley AJ: RhoE regulates actin cytoskeleton organization and cell migration. Mol Cell Biol 18: 4761-4771, 1998.

8. Bektic J, Pfeil K, Berger AP, et al: Small G-protein RhoE is underexpressed in prostate cancer and induces cell cycle arrest and apoptosis. Prostate 64: 332-340, 2005.

9. Poch E, Miñambres R, Mocholí E, et al: RhoE interferes with $\mathrm{Rb}$ inactivation and regulates the proliferation and survival of the U87 human glioblastoma cell line. Exp Cell Res 313: 719-731, 2007.

10. Villalonga P, Guasch RM, Riento K and Ridley AJ: RhoE inhibits cell cycle progression and Ras-induced transformation. Mol Cell Biol 24: 7829-7840, 2004.

11. Zhu YJ, Shi F, Zhang SY, et al: Expression of RhoE in gastrointestinal cancer and its clinical significance. Mod Oncol (Chin) 16: 989-992, 2008

12. Huang $M$ and Prendergast GC: RhoB in cancer suppression. Histol Histopathol 21: 213-218, 2006.

13. Mazières $\mathrm{J}$, Tovar $\mathrm{D}, \mathrm{He} \mathrm{B}$, et al: Epigenetic regulation of RhoB loss of expression in lung cancer. BMC Cancer 7: 220-227, 2007.

14. Nishigaki M, Aoyagi K, Danjoh I, et al: Discovery of aberrant expression of R-RAS by cancer-linked DNA hypomethylation in gastric cancer using microarrays. Cancer Res 65: 2115-2124, 2005.

15. O'Hagan KA, Cocchiglia S, Zhdanov AV, et al: PGC-1alpha is coupled to HIF-1alpha-dependent gene expression by increasing mitochondrial oxygen consumption in skeletal muscle cells. Proc Natl Acad Sci USA 106: 2188-2193, 2009.

16. Wang S, Yan-Neale Y, Fischer D, et al: Histone deacetylase 1 represses the small GTPase RhoB expression in human nonsmall lung carcinoma cell line. Oncogene 22: 6204-6213, 2003.

17. Livak KJ and Schmittgen TD: Analysis of relative gene expression data using real-time quantitative PCR and the 2(-Delta Delta C (T)) method. Methods 25: 402-408, 2001.

18. Ongusaha PP, Kim HG, Boswell SA, et al: RhoE is a prosurvival p53 target gene that inhibits ROCK I-mediated apoptosis in response to genotoxic stress. Curr Biol 16: 2466-2472, 2006.

19. Gress TM, Müller-Pillasch F, Geng M, et al: A pancreatic cancer-specific expression profile. Oncogene 13: 1819-1830, 1996.
20. Zhang C, Zhou F, Li N, et al: Overexpression of RhoE has a prognostic value in non-small cell lung cancer. Ann Surg Oncol 14: 2628-2635, 2007.

21. Boswell SA, Ongusaha PP, Nghiem P and Lee SW: The protective role of a small GTPase RhoE against UVB-induced DNA damage in keratinocytes. J Biol Chem 282: 4850-4858, 2007.

22. Hansen SH, Zegers MM, Woodrow M, Rodriguez-Viciana P, Chardin P, Mostov KE and McMahon M: Induced expression of Rnd3 is associated with transformation of polarized epithelial cells by the Raf-MEK-extracellular signal-regulated kinase pathway. Mol Cell Biol 20: 9364-9375, 2000.

23. Riento K, Guasch RM, Garg R, Jin B and Ridley AJ: RhoE binds to ROCK I and inhibits downstream signaling. Mol Cell Biol 23: 4219-4229, 2003

24. Tanimura S, Nomura K, Ozaki K, Tsujimoto M, Kondo T and Kohno M: Prolonged nuclear retention of activated extracellular signal-regulated kinase $1 / 2$ is required for hepatocyte growth factor-induced cell motility. J Biol Chem 277: 28256-28264, 2002.

25. Riento K, Totty N, Villalonga P, Garg R, Guasch R and Ridley AJ: RhoE function is regulated by ROCK I-mediated phosphorylation. EMBO J 24: 1170-1180, 2005.

26. Bektic J, Wrulich OA, Dobler G, et al: Identification of genes involved in estrogenic action in the human prostate using microarray analysis. Genomics 83: 34-44, 2004.

27. Liu T, Bauskin AR, Zaunders J, Brown DA, Pankhurst S, Russell PJ and Breit SN: Macrophage inhibitory cytokine 1 reduces cell adhesion and induces apoptosis in prostate cancer cells. Cancer Res 63: 5034-5040, 2003.

28. Esteller M: Epigenetics in cancer. N Engl J Med 358: 1148-1159, 2008.

29. Choi IS and Wu TT: Epigenetic alterations in gastric carcinogenesis. Cell Res 15: 247-254, 2005.

30. Nardone G and Compare D: Epigenetic alterations due to diet and Helicobacter pylori infection in gastric carcinogenesis. Expert Rev Gastroenterol Hepatol 2: 243-248, 2008.

31. Cameron EE, Bachman KE, Myöhänen S, Herman JG and Baylin SB: Synergy of demethylation and histone deacetylase inhibition in the re-expression of genes silenced in cancer. Nat Genet 21: 103-107, 1999.

32. Pfeifer GP and Dammann R: Methylation of the tumor suppressor gene RASSF1A in human tumors. Biochemistry (Mosc) 70: 576-583, 2005.

33. Pong RC, Lai YJ, Chen H, Okegawa T, Frenkel E, Sagalowsky A and Hsieh JT: Epigenetic regulation of coxsackie and adenovirus receptor (CAR) gene promoter in urogenital cancer cells. Cancer Res 63: 8680-8686, 2003. 